\title{
CARACTERIZACION DEL ACEITE ESENCIAL EN LA CORTEZA DEL LIMON SWINGLEA (Swinglea glutinosa) POR CG/EM
}

\section{CHARACTERIZATION OF ESSENTIAL OIL OF SWINGLEA LEMON (Swinglea glutinosa) PEEL FOR GC-ME}

\author{
Carlos Díaz¹, Guillermo Arrázola², Fabián Ortega³ , Jair Gaviria ${ }^{1}$
}

\begin{abstract}
RESUMEN
Para la extracción y análisis del aceite esencial de la corteza del limón Swinglea glutinosa se escogieron frutos con un índice de madurez comprendido entre 3.73 y 8.83. Para la obtención del extracto fue necesario raspar la cáscara, solubilizarla en una solución de etanol al 5\% e hidrodestilar por 2 horas, obteniéndose rendimientos de extracción entre 0.20 y $0.39 \%$. Los constituyentes volátiles se analizaron mediante Cromatografía de Gases acoplada a Espectrometría de Masas (CG-EM). Se identificaron 14 componentes dentro de los cuales se destacaron por su abundancia $\beta$-Cubebeno (26.48-28.74\%), $\beta$ Pineno (24.19-28.83\%), Elixeno (10.13-11.03\%), $\beta$-Cariofileno (6.30-9.55\%), $\beta$-Felandreno (5.99-7.86\%), a-Pineno (4.44-6.67\%), D-Limoneno (4.45-5.38\%) y Anozol (1.45-4.38\%). La identificación de los constituyentes se efectuó mediante la comparación de los espectros de masa obtenidos con los de una base datos controlada por computador (NIST 98 versión 2.0) y algunos compuestos mediante la comparación de los tiempos de retención con muestras puras en las mismas condiciones experimentales.
\end{abstract}

Palabras claves: Cromatografía de gases, espectrometría de masas, hidrodestilación, limoneno.

\begin{abstract}
For the extraction and analysis of the essential oil of the peel of Swinglea glutinosa lemon fruits were chosen with a maturity index between 3.73 and 8.83 . The peel was processed, mix et with ethanol solution ( $5 \%$ ) and hydro-distillate et for 2 hours; yields between 0.20 and $0.39 \%$ were obtained. The volatile constituents were analyzed by means of Gas Chromatography and Mass Spectrometry (GC-MS). 14 components were identified among them $\beta$-Cubebene (26.48-28.74\%), $\beta$-Pinene $(24.19-28.83 \%)$, Elixene (10.13-11.03\%), $\beta$-Caryophillene (6.30-9.55\%), $\beta$-Phellandrene (5.99-7.86\%), $\alpha$-Pinene (4.44$6.67 \%)$, D-Limonene (4.45-5.38\%) and Anozol (1.45-4.38\%) were abundant. The identification of some of the constituents was done by mass spectra comparison with those of the database (NIST 98 version 2.0) and others by matching the retention times with pure samples under the same experimental conditions.
\end{abstract}

Keywords: gas chromatography, mass spectrometry, hydrodistillation, limonene.

\footnotetext{
${ }^{1}$ Ingeniero de Alimentos.

${ }^{2}$ Ingeniero de Alimentos. Ph.D. Universidad de Córdoba. Montería Colombia.

Email: garrazola@sinu.unicordoba.edu.co

${ }^{3}$ Ingeniero de Alimentos. Departamento de Ingenieria de Alimentos. Universidad de Córdoba
} 


\section{INTRODUCCION}

En las distintas zonas agroclimáticas de Colombia crece gran variedad de especies herbáceas y arbustivas, de las cuales se pueden extraer diversas sustancias, tales como taninos, saponina y aceites esenciales. En nuestro país los estudios realizados sobre estos recursos, sus principios activos, posibilidades de industrialización, usos potenciales y mercadeo de los mismos se encuentra en fase exploratoria comparados con otros países, lo cual ha impedido explotar en forma industrial muchas especies propias de nuestra gran biodiversidad (Velásquez y Zuluaga, 1997).

En Colombia se reportan aproximadamente 50000 especies de flora, ésto representa una gran oportunidad para nuestro país en el mercado de los productos forestales no maderables como: aceites esenciales y oleorresinas, gomas y resinas, colorantes, pigmentos y tintas naturales, hierbas $y$ especias, plantas medicinales y fitofarmaceúticos; no obstante, existen muchas especies que no son aprovechadas industrialmente, dentro de éstas se encuentra el árbol del limón Swinglea, el cual se adapta a las condiciones agroecológicas del departamento de Córdoba. Este árbol se usa en nuestra región como ornamental, para cerca viva y como filtro de olores en lagunas de oxidación (Pérez, 1956); recientemente de la corteza de ésta especie se han aislado alcaloides (acridonas) con actividad antimalárica (Weniger, 2001), lo mismo que alcaloides, triterpenos y acetatos del extracto de la fruta, aplicando técnicas de análisis instrumental tales como espectroscopia y resonancia magnética nuclear (Dreyer, 1970).

La mayoría de los aceites esenciales cítricos han sido estudiados (naranja, pomelo, limón, mandarina, etc.) y se ha determinado su composición química lo cual ha permitido establecer usos y valor comercial de los mismos; los aceites cítricos constituyen el sector más grande del la producción mundial de aceites esenciales. La alta concentración de compuestos como citronelal, linalol y citral hacen que estos aceites sean muy atractivos para la industria del aroma y sabor (Blanco et al., 1995). El limón Swinglea dentro de su clasificación taxonómica es un cítrico, sin embargo, es limitada la información que se ha publicado acerca del mismo tales como aspectos agronómicos, composición química, usos industriales, etc. A diferencia de Colombia, en otros países la industria de los aceites esenciales está muy desarrollada y encuentra gran aplicación en la medicina, la industria cosmética, de alimentos y de variados productos obtenidos mediante la industria química. Sin embargo, nuestro país a pesar de su potencial en biodiversidad es un gran importador de este tipo de productos (Velásquez y Zuluaga, 1997). Este trabajo de investigación tuvo como objetivo extraer y determinar la composición del aceite esencial de la corteza del limón Swinglea por cromatografía de gases / espectrometría de masas (CG-EM), lo cual permitirá posteriormente a través de estudios complementarios establecer la calidad, el valor comercial y los posibles usos industriales del mismo.

\section{MATERIALES Y METODOS}

\section{Determinación de los constituyentes volátiles de la cáscara}

Para la determinación de los componentes volátiles del aceite esencial de la cáscara del limón Swinglea se clasificó el fruto según el índice de madurez. Las muestras fueron recolectadas de árboles de cercas vivas de la Universidad de Córdoba sede Berástegui y de la empresa Diagonal, situadas en el Km 16 vía Ciénaga de Oro. Para la clasificación del fruto de acuerdo al estado de madurez se tomaron cincuenta (50) muestras al azar en distintos árboles y se agruparon en tres estados subjetivamente de manera a priori de acuerdo al desarrollo de sus características 
como color, tamaño, textura, codificándolos en tres estados de madurez (E1, E2 y E3). A cada muestra se le evaluaron los siguientes parámetros por triplicado: sólidos solubles totales, pH, acidez total, color de la corteza, altura y diámetro del fruto, peso del fruto. Los patrones puros y los reactivos para cromatografía fueron marca Sigma ${ }^{\circledR}$ y los solventes utilizados de marca Carlos Erba ${ }^{\circledR}$.

Para la extracción del aceite esencial se seleccionaron separadamente frutos en los estados de madurez E1, E2 y E3, una vez descortezados se pesaron $200 \mathrm{~g}$ de la corteza obtenida y se mezcló con un solvente en agua destilada, relación corteza / solución: 1/8 (P/V) (Correa et al. 1992); para la selección del solvente se hicieron pruebas con diferentes compuestos (hexano, éter de petróleo y etanol) observando el comportamiento de la sustancia glutinosa en cuanto a su solubilidad. Una vez seleccionado el solvente se hicieron ensayos variando la concentración del mismo (2, 5, 10 y $20 \%$ ) para determinar su influencia en el rendimiento de extracción; la mezcla obtenida se sometió a hidrodestilación por 2 horas a presión atmosférica. El destilado obtenido se decantó por 1 hora y posteriormente se separó el aceite esencial para centrifugarlo por 5 minutos a 2000 r.p.m. Finalmente las muestras se sometieron a congelación $\left(-20^{\circ} \mathrm{C}\right)$ para eliminar la humedad restante y se conservaron bajo estas mismas condiciones hasta la realización de los análisis cromatográficos. La experimentación anterior fue adaptada del trabajo de Blanco et al. (2004). Los análisis cromatográficos se realizaron por triplicado para cada estado de madurez y las muestras de aceite se diluyeron en diclorometano (100 $\mathrm{mg}$ de aceite por cada $\mathrm{ml}$ de solvente).

\section{Condiciones cromatográficas}

Las muestras previamente acondicionadas fueron inyectadas a un cromatógrafo de gases (Agilent Technologies $6890 \mathrm{~N}$ ), acoplado a un espectrómetro de masas (Agilent 5973 Network) con las siguientes condiciones de trabajo: Columna (HP-5, longitud $30 \mathrm{~m}$, diámetro $0.25 \mathrm{~mm}$, espesor de la fase estacionaria $0.25 \mathrm{~mm}$, gas portador Helio, velocidad lineal de $30 \mathrm{~cm}^{-1}$, inyección de split 50:1), programación de temperatura del horno de la columna $3 \mathrm{~min}$ a $50^{\circ} \mathrm{C}, 50-250^{\circ} \mathrm{C}$ a $30^{\circ} \mathrm{C} \mathrm{min} \mathrm{m}^{-1}, 250-320^{\circ} \mathrm{C}$ a $25^{\circ} \mathrm{C} \mathrm{min}^{-1}$, temperatura del inyector de $200^{\circ} \mathrm{C}$, temperatura interfase del detector de $260^{\circ} \mathrm{C}$.

La identificación de los componentes se efectuó mediante la comparación de los espectros de masa obtenidos con los de la base de datos NIST 98 Mass Spectral Library, (versión 2.0), y algunos compuestos mediante la comparación de los tiempos de retención con los de muestras puras, en las mismas condiciones experimentales. El análisis de resultados se evaluó con un análisis de varianza y prueba de comparación de medias con el software estadístico STATGRAPHICS PLUS 5.0 con un $95 \%$ de confiabilidad.

\section{RESULTADOS Y DISCUSION}

\section{Constituyentes volátiles de la corteza} Los frutos escogidos mostraron un índice de madurez promedio comprendido entre 3.73 y 8.83, (Tabla 1).

Tabla 1. Clasificación del fruto de acuerdo al estado de madurez.

\begin{tabular}{lccc}
\hline Propiedades & Estado de madurez 1 & Estado de madurez 2 & Estado de madurez 3 \\
\hline \% Acido cítrico $(\mathrm{p} / \mathrm{v})$ & $3.98( \pm 0.427)$ & $3.36( \pm 0.806)$ & $2.79( \pm 0.821)$ \\
${ }^{\circ}$ Brix corregidos & $14.56( \pm 1.295)$ & $18.11( \pm 4.928)$ & $21.69( \pm 5.165)$ \\
Indice de madurez & $3.73( \pm 0.75)$ & $6.02( \pm 3.60)$ & $8.83( \pm 4.01)$ \\
\hline
\end{tabular}


En el análisis de varianza se encontraron diferencias significativas $(P r<0.05)$ entre los tres estados de madurez elegidos.

Para obtener los extractos de la corteza no fue posible emplear el método de expresión en frío directamente, dicho comportamiento se explica debido a que el Limón Swinglea posee una sustancia glutinosa (pegante) en la pulpa y en la corteza que impide la separación del aceite contenido en las glándulas del flavedo del fruto. Tampoco fue posible extraer el aceite esencial destilando la corteza en una solución acuosa. Finalmente se obtuvo resultados satisfactorios solubilizando las muestras de corteza en una solución de alcohol etílico al 5\% y posteriormente empleando el método de arrastre con vapor (hidrodestilación). Es importante anotar que la sustancia glutinosa de la corteza del limón swinglea es insoluble en otros solventes como hexano y éter de petróleo y que además para soluciones alcohólicas con concentraciones por encima y por debajo del 5\%, los rendimientos de extracción son bajos. Este comportamiento se puede atribuir a que con concentraciones de alcohol por debajo del 5\%, no se logra solubilizar totalmente la sustancia glutinosa que impide la separación del aceite esencial. De otro lado, cuando la concentración es superior al 5\% los rendimientos bajan debido a que el aceite esencial se solubiliza en el etanol en exceso.

La tabla 2 muestra el rendimiento de extracción del aceite esencial del fruto medido con respecto al peso de la corteza.

Tabla 2. Rendimientos de extracción del aceite esencial de la corteza.

\begin{tabular}{lccc}
\hline & Estado de madurez 1 & Estado de madurez 2 & Estado de madurez 3 \\
\hline Muestras $(\mathrm{n}):$ & 3 & 3 & 3 \\
Promedio $\%(\mathrm{p} / \mathrm{p}):$ & $0.20 \pm 0.03$ & $0.26 \pm 0.01$ & $0.39 \pm 0.03$ \\
\hline
\end{tabular}

Se observa que el mayor rendimiento de extracción se obtiene para frutas en estado 3, lo cual demuestra que el estado de madurez tiene una marcada influencia sobre los rendimientos de extracción. El anova mostró la existencia de diferencias significativas $(P r<0.05)$ en el rendimiento de extracción de aceite esencial entre los tres estados de madurez de los frutos estudiados. Los rendimientos de extracción obtenidos para el caso del limón Swinglea son mayores a los reportados por Blanco et al. (1995), quienes extrajeron el aceite esencial de la corteza del limón (Citrus volkameriana) por arrastre con vapor y obtuvieron un rendimiento promedio de $0.19 \%(\% \mathrm{P} / \mathrm{P})$. Autores como Sawanura et al. (2004) y Stashenko et al. (1996) muestran resultados en la extracción de aceites como limoneno con $94 \%$, $\alpha$-pineno $54 \%$, mirceno con un
$1.2 \%$, todos excepto el limoneno son mucho menores al obtenido en el limón Swinglea.

El resultado de los análisis cromatográficos realizados al aceite esencial extraído de la corteza del Limón Swinglea se muestran en la tabla 3. Se observa un comportamiento similar en la composición de volátiles de los aceites extraídos de la corteza de los estados de madurez E1, E2 y E3, respectivamente. Estos resultados muestran la presencia de ocho componentes ( $\beta$-Cubebeno, $\beta$-Pineno, Elixeno, Anozol, $\beta$-Cariofileno, $\beta$-Felandreno, D-Limoneno y $\alpha$-Pineno) en todas las muestras analizadas, los cuales debido a su abundancia se pueden considerar como mayoritarios, destacándose el $\beta$-Cubebeno (26.48-28.74\%), el $\beta$-Pineno (24.19-28.83\%) y el Elixeno (10.13-11.03\%). El anova realizado para cada uno de estos compuestos 
en el cual se evalúa la composición química del aceite esencial en función del estado de madurez del fruto permite afirmar que ésta variable no afecta significativamente $(P r>0.05)$ la composición en volátiles del aceite esencial. Los mismos compuestos encontrados presentan características iguales para los diferentes estados de maduración, como por ejemplo, tiempo de retención.

Tabla 3. Composición del aceite esencial de la corteza en estados E1, E2 y E3

\begin{tabular}{|c|c|c|c|c|c|}
\hline & \multirow[t]{2}{*}{ Identificación } & \multirow[t]{2}{*}{$\begin{array}{c}\text { Tiempo retención } \\
\text { (minutos) }\end{array}$} & \multirow{2}{*}{$\begin{array}{c}\text { Abundancia } \\
(\% \mathrm{p} / \mathrm{p}) \\
\text { E1 }\end{array}$} & \multirow{2}{*}{$\begin{array}{c}\text { Abundancia } \\
(\% \mathrm{p} / \mathrm{p}) \\
\text { E2 }\end{array}$} & \multirow{2}{*}{$\begin{array}{c}\text { Abundancia } \\
\text { (\% p/p) } \\
\text { E3 }\end{array}$} \\
\hline & & & & & \\
\hline 1 & $\beta$-Cubebeno & 8,940 & 28.741 & 26.485 & 28.838 \\
\hline 2 & $\beta$-Pineno & 6.050 & 24.195 & 25.891 & 27.753 \\
\hline 3 & Elixeno & 9.168 & 11.039 & 10.591 & 10.137 \\
\hline 4 & Anozol & 9.320 & 7.385 & 4.076 & 4.389 \\
\hline 5 & $\beta$-Cariofileno & 8,670 & 6.307 & 9.553 & 6.937 \\
\hline 6 & $\beta$-Felandreno & 6.009 & 5.993 & 6.862 & 7.865 \\
\hline 7 & D-Limoneno & 6.430 & 4.452 & 4.772 & 5.389 \\
\hline 8 & $\alpha$-Pineno & 5.660 & 4.441 & 5.704 & 6.673 \\
\hline 9 & $\tau$-Selineno & 9.019 & 1.404 & 1.407 & - \\
\hline 10 & $\alpha$-Cariofileno & 8,826 & 1.280 & 1.510 & - \\
\hline 11 & $\gamma$-Terpineno & 6.636 & 1.160 & 0.951 & 1.271 \\
\hline 12 & $\delta$-Elemeno & 8,232 & 0.914 & 0.694 & - \\
\hline 13 & Cyclogeraniolano & 4,661 & 0.661 & - & - \\
\hline 14 & $\alpha$-Cadinol & 9.635 & 0.513 & - & - \\
\hline 15 & Elixeno & 9.168 & 10.553 & - & - \\
\hline 16 & $\begin{array}{l}\text { Hexadecano 1,1 } \\
\text { bis(dodecyloxi) }\end{array}$ & 4.623 & 0.825 & 0.825 & - \\
\hline
\end{tabular}

Los resultados permiten afirmar que la composición del aceite esencial de la corteza del limón Swinglea difiere significativamente con respecto a la mayoría de cítricos hasta el momento estudiados y explotados comercialmente, ya que en estos usualmente el componente mayoritario suele ser el DLimoneno. Esta afirmación se puede corroborar por varios autores que han estudiado otras variedades de frutas cítricas, como Braddock (1999) quien afirma que el mayor constituyente del aceite de la cáscara de los cítricos comestibles es el D-Limoneno. Otra investigación que constata la afirmación hecha por el anterior autor fue hecha por Lota et al. (2002), quienes estudiaron la composición del aceite esencial de la corteza de 19 variedades de limón por el método de expresión en frío y posterior análisis por CGEM, identificando 51 componentes en todos los aceites esenciales analizados, y donde el Limoneno presentó la mayor abundancia (38.1-95.8\%), seguido por el $\beta$-Pineno $(0.1-$ $15.8 \%), \gamma$-Terpineno (trazas-18\%), linalol $(0.1-25.1 \%)$ y acetato de linalilo (trazas$31.2 \%)$.

Para el caso del limón Swinglea el Dlimoneno se encuentra presente en baja proporción (4.45-5.39\%) con respecto a otras especies y variedades de cítricos (Stashenko, 1996); sin embargo, éste muestra una 
presencia significativa de otros componentes como el $\beta$-Pineno $(24.2-28.84 \%$ ) y el $\beta$ Cubebeno (26.48-28.74\%). Este comportamiento puede tener explicación en el hecho de que de una especie a otra se pueden presentar diferencias significativas en la composición de volátiles por factores genéticos y ambientales.

Uno de los aspectos relevantes en la composición del aceite esencial de la cáscara del limón Swinglea es la proporción en la que se identificó el $\beta$-Pineno (24.20-28.84\%) y el $\beta$-Cubebeno (26.48-28.74\%). Ninguno de los autores citados anteriormente reportan el $\beta$-Pineno con abundancias por encima del $16 \%$. En efecto, Blanco et al. (1995), reportan porcentajes de $\beta$-Pineno que varían entre 0.29 y $1.05 \%$ para limón, mandarina y naranja. El $\beta$-Cubebeno ha sido reportado para algunas variedades de cítricos, pero en muy baja proporción. Minh et al. (2002), identificó el $\beta$-Cubebeno en una proporción de $0.1 \%$, en el aceite esencial de la corteza de Citrus shaerocarpa (una de las frutas cítricas más populares en el Japón), por el método de expresión en frío y análisis CGEM. Caccioni (1998), identificó el $\beta$ Cubebeno por CG-EM en el aceite esencial extraído por arrastre con vapor de la corteza de Citrus aurantium (0.05\%), $0.06 \%$ en Citrus sinensis $x$ Poncirus trifoliata (Carrizo citrange) y $0.3 \%$ en Citrus sinensis x Poncirus trifoliata (citrange Troyer). Otro aspecto importante es la presencia de compuestos como Elixeno, $\beta$-Cariofileno, $\beta$-Felandreno, $\alpha$-Pineno y Anozol en proporciones que varían entre 4 y $11 \%$, normalmente éstos compuestos se encuentran en baja proporción en la mayoría de los cítricos de interés comercial. Minh et al. (2002), reportan el $\beta$-Cariofileno $(0.2 \%)$ y $\alpha$-Pineno $(0.7 \%)$; Lota et al. (2002), reportan el $\beta$ Felandreno $(0.1-4.2 \%)$ y el $\alpha$-Pineno $(0.1-$ $2.3 \%)$; Caccioni et al. (1998), identificaron el $\beta$-Felandreno $(0.03-0.52 \%)$, $\alpha$-Pineno $(0.4-$ $2.27 \%), \beta$-Cariofileno $(0.01-1.6 \%)$ en diferentes cítricos procedentes de Italia (naranjas, mandarinas, limones y algunos híbridos). No se encontraron reportes de Elixeno y Anozol en cítricos.

\section{CONCLUSIONES}

- En los rendimientos en extracción del aceite esencial de la corteza se obtienen resultados satisfactorios cuando el etanol una concentración del $5 \%$ donde el estado de madurez influye significativamente. El método en frío no es aplicable para este caso.

- Los componentes mayoritarios del aceite esencial de la corteza en los estados de madurez E1 y E2 son en su orden $\beta$ Cubebeno, $\beta$-Pineno y Elixeno, en el estado E3 el $\beta$-Pineno, $\beta$-Cubebeno y Elixeno.

- La composición química del aceite esencial de corteza muestra la ausencia de compuestos oxigenados (aldehídos, cetonas, esteres y alcoholes) como neral, citral, geraniol, linalol y citronelal, lo cual hace que el aroma no sea el característico de los cítricos.

- El limón Swinglea posee un alto potencial para la extracción de compuestos utilizados en la industria alimentaria, tales como: $\beta$-Pineno, $\beta$-Cubebeno, $\beta$ Cariofileno.

\section{AGRADECIMIENTOS}

Los autores expresan sus agradecimientos a la Universidad de Córdoba, en especial al personal del laboratorio de análisis de alimentos y al CEINA; al personal del laboratorio de análisis instrumental de la Universidad Nacional con sede en Medellín, y a todas las personas que hicieron posible la realización de este proyecto de investigación. 


\section{BIBLIOGRAFIA}

Blanco, C.; Grane, N. y Nieto, M. 1995. Comparative study of Colombian citrus oils by high-resolution gas chromatography and gas chromatography-mass spectrometry. Journal of Chromatography 697:501 $-513$.

Braddock, R. 1999. Handbook of Citrus. Products and Processing Technology. Editorial Wiley, New York, p.247256.

Caccioni M. 1998. Relationship between volatile components of citrus fruit essential oils and antimicrobial action on Penicillium digitatum and Penicillium italicum. International Journal of Food Microbiology 43:7379

Correa, G.; Gómez, M. y Rojo, A. 1999. Desarrollo experimental de una unidad piloto para la extracción de aceite esencial de manzanilla. Tesis Ingeniero Químico, Universidad Pontificia Bolivariana (Medellín), 149 p.

Dreyer, D. 1970. Extractives of Swinglea glutinosa (bl.) Merr. Tetrahedron Journal 26 (24):5745-5751.

Lota, M.; Serra R.; Tomi, F. y Casanova, J. 2002. Variability of peel and leaf essential oils from Citrus reticulata Blanco. Journal Biochemical Systematic and Ecology Chemical 28:61-78
Minh, T.; Li, P. y Tao, N. 2002. Characteristic odor components of Citrus Shaerocarpa Tanaka (Kabosu) coldpressed peel oil. Journal of Agricultural and Food Chemistry 50:2908-2913.

Pérez, E. 1956. Plantas útiles de Colombia. Camacho Roldan Ltda, Bogota, 590p.

Stashenko, E.; Martínez, R.; Pinzón, H. y Ramírez. J. 1996. Journal of Chromatography A, 752:217-222

Sawanura, M.; Son, U.; Choi H.; Kim, M.; Phi, N.; Fears, M. y Kumagai, C. 2004. Compositional changes in commercial lemon essential oil for aromatherapy. International Journal of Aromatherapy, 14:346-368

Velasquez, E. y Zuluaga, O. 1997. Diseño de un proceso industrial para la obtención de aceites esenciales de dos especies nativas colombianas bajo la filosofía de cero emisiones. Tesis Ingeniero Industrial, Universidad EAFIT, Medellín, 178p

Weniger, M. 2001. Bioactive Acridone Alkaloids from Swinglea glutinosa. Journal of Natural Products 64:12211223 